\title{
DYNAMICAL HEAT-FLUX FEEDBACKS AND GLOBAL
}

\author{
CLIMATE STABILITY
}

\author{
by \\ Gyula Molnar and Wei-Chyung Wang \\ (Atmospheric and Environmental Research Inc., 840 Memorial Drive, Cambridge, \\ Massachusetts 02139, U.S.A.)
}

\section{ABST RACT}

In the face of the faint young sun-climate paradox, the plausible effects of dynamical heat-flux feedbacks on global stability were examined using a coupled high- and low-latitude radiative-dynamical model. The global stability is found to depend very strongly on the vertical heat transport, which is also closely coupled to the meridional heat transport. This coupling and the associated dynamical heat-flux feedbacks, treated with in the framework of the radiative-dynamical model, was found to produce a fairly strong negative feedback. Our results indicate that the dynamical heat-flux feedbacks inherent in the climate system may potentially enhance the global stability.

\section{INTRODUCTION}

According to theories of stellar evolution (see, for example, Newk irk (1980) for a comprehensive review), the Sun was initially much fainter than it is at the present time. The solar luminosity of $4.7 \mathrm{Ga} B P$ was calculated to be about $71 \%$ of the present value, increasing almost linearly since then (Endal and Schatten 1982). It would appear that a decrease of the present value of solar luminosity by 20 to $30 \%$ could initiate an ice age and result in a totally icecovered Earth (cf., for example, North and others 1981).

However, the geological record has indicated that the recent climate is more glacial than the long-term average (cf. Tarling 1978, Crowley 1983). This would imply that, during most of the Earth's history, the mean surface temperature has been near or above the present value. It also suggests that the Earth must have resisted complete glaciation during its evolution, al though widespread glaciations were evident even in early climate history (e.g. the Huronian glaciation $\sim 2.3 \mathrm{Ga} \mathrm{BP}$ ). To complicate the picture further, there seems to be a decrease in the Earth's surface temperature over the past $3 \mathrm{Ga}$ (Knauth and Epstein 1976), inferred from the isotopic analys is of cherts. This contradiction between the timedependent solar output and the mean surface temperature of the Earth has been termed the "faint young sun-cl imate paradox".

In studying the problem, it is more convenient to refer to the term "global stability" (cf. Lindzen and Farrel1 1977), defined as the percent decrease of the present solar constant $S_{0}$ so that a totally ice-covered Earth occurs. Thus, the global stability represents the ability for the Earth- atmosphere climate system to resist complete glaciation in the face of a significantly reduced $S_{0}$. Past studies of the global stability have been 1 imited to the use of the one-dimensional zonal energy balance (ZEB) models. Budyko (1969) and Sellers (1969) have indicated that the global stability is small, about $2 \%$. Using different meridional dynamical heat-flux parameterizations, North (1975) and Lindzen and Farrell (1977) obtained values of about 4 and $7 \%$, respectively; Lindzen and Farrell also computed a value of $17 \%$ with an ad hoc $\mathrm{Hadley}$ stability ledge. More recent models (Coakley 1979, Warren and Schneider 1979) with improved parameterizations for the outgoing infrared (IR) flux and solar albedo yielded a value of about $10 \%$. Warren and Schneider have also shown that the difference in the IR flux and albedo parameterizations could yield different global stability, ranging from 3 to $21 \%$. On the other hand, using a one-dimensional vertical radiative-convective (VRC) model with ice albedo-temperature feedback and more comprehensive radiation parameterizations, Wang and S tone (1980) computed the global stability to be about 11 to $23 \%$, depending on the treatment of clouds.

Hypotheses have been proposed to explain the faint young sun-climate paradox. Sagan and Mullen (1972) and Hart (1978) discussed the possibility of the atmospheric greenhouse effect, associated with the chemical evolution of the Earth-atmosphere system. However, Owen and others (1979), Kuhn and Atreya (1979), and Kasting (1982) have argued against the ammonia- and methane-based greenhouse scenarios, primarily because the importance of these trace gases was limited to a small fraction of the Earth's history and thus only $\mathrm{H}_{2} \mathrm{O}$ and $\mathrm{CO}_{2}$ could be possible candidates. A review of other possible hypotheses can be found in the work of Endal and Schatten (1982).

Here, we show that the cl imate feedback mechanisms, especially the vertical and meridional dynamical heat fluxes and their interactions, could act to maintain a much more stable climate. In particular, we use a coupled high- and low-latitude radiativedynamical (HLRD) model to demonstrate the strength of such a strong $\mathrm{cl}$ imate-stabilizing effect. However, it must be borne in mind that the uncertainty associated with the dynamical heat-flux parameterizations is large, especially when used in a drastically different (from the present) climate. Nevertheless, our purpose is to demonstrate the role of such a plausible climate-stabilizing mechanism. Below, we will first describe the physical model which includes these feedbacks. The model results of 
the effect of dynamical heat-flux feedbacks on global stability will be presented in section 3 . In section 4 , we summarize our $f$ indings.

2. MDDEL

The coupled HLRD model is constructed to compute the annual mean northern-hemispheric temperature distribution $T(x, z)$ as a function of altitude $z$ and latitude $\phi$ where $x=\sin \phi$. The vertical and meridional temperature structure is determined from heat balance between radiation and dynamical heat fluxes.

We use high vertical resolution (similar to the VRC model) because, as shown in Wang and others (1980, $1981)$, the change in vertical distribution of atmospheric trace constituents and clouds could have an important radiative effect on model climate sensitivity. However, very low-latitudinal resolution is chosen: two latitude zones are considered, i.e. a low latitude $\left(0\right.$ to $\left.30^{\circ} \mathrm{N}\right)$ and a mid-to-high latitude ( 30 to $\left.90^{\circ} \mathrm{N}\right)$. Note that the low-latitudinal resolution will limit the study to the effect of dynamical heat fluxes on climate sensitivity on an annual basis. By using a two-zone model, the spatial scales are sufficiently large for one to anticipate that forced variations will dominate over free variations (Lorenz 1979). In addition, we minimize the complex problem of parameterizing the oceanic heat flux in low latitudes. Furthermore, as noted by North and others (1981), a two-zone latitudinal resolution is necessary and sufficient for using a two-mode Legendre polynomial expansion of meteorological fields (see discussion below). The two-zone resolution also allows for the moist adiabatic adjustment in the tropical region as well as for the baroclinic adjustment in high latitudes, which is a very important feature of the model climate system.

To represent the annual mean northern-hemispheric distribution of solar radiation reaching the top of the atmosphere $S(x)$ and the surface temperature $T_{S}(x)$, we use a two-mode representation (North 1975):

$$
\begin{aligned}
& S(x)=\left[1+S_{2} P_{2}(x)\right] \bar{S} \\
& T_{S}(x)=\bar{T}_{S}+T_{2} P_{2}(x)
\end{aligned}
$$

where $P_{2}(x)$ is the second-order Legendre polynomial, $\bar{S}$ the mean value of $S(x)$ and $S_{2}=-0.477$ (North and others 1981). The northern-hemispheric mean surface temperature $\bar{T}_{s}$ and the effective meridional temperature contrast $T_{2}$ are the primary unknowns of the model.

In the HLRD model, the solar radiation is based on Lacis and Hansen (1974) while the thermal radiation formulation has been described in detail by Wang (1983). Spectral radiative properties of clouds were included in both the solar and thermal radiation calculations.

We use the empirical expression derived by Stone and Miller (1980) to relate the total meridional heat transport (MHT) to the meridional surface temperature gradient. Specifically, from their equations, we find the total meridional heat flux $D$

$$
\mathrm{D}\left(30^{\circ} \mathrm{N}\right)=3.25\left(-\mathrm{T}_{2}-12.32\right) \times 10^{14} \mathrm{~W} .
$$

The vertical dynamical heat flux is simulated by convective adjustment (Manabe and Strickler 1964). Following Stone and Carlson (1979), the critical lapse rate is chosen to be the moist adiabate $\Gamma_{m}$ for the equatorial zone and at the 30 to $90^{\circ} \mathrm{N}$ zone it is constrained by both $\Gamma_{m}$ and $\Gamma_{b}$ (the critical value for baroclinic adjustment). Note that, implicitly, $\mathrm{r}_{\mathrm{b}}$ depends on the low-latitude $\Gamma_{m}$ through its dependence on the meridional temperature gradient.

The ice albedo-temperature parameterization is similar to the one used by Wang and Stone (1980). In the HLRD model, it is necessary to parameterize the change of the zonal surface albedo as a function of the ice-line change, which in turn depends on the changes in the zonal temperature distribution, i.e. in the two-mode representation $T_{s}$ and $T_{2}$. The highlatitude surface albedo $\alpha_{\mathrm{SH}}$ can be calculated from the following expression:

$$
a_{S H}=\frac{a_{H}\left(x_{S}-0.5\right)+b\left(1-x_{S}\right)+s_{2}\left[3 a_{H} / 16+\left(b-a_{H}\right)\left(x_{S}-x_{S}{ }^{3}\right) / 2\right]}{0.5+3 s_{2} / 16}
$$

Whenever the ice-line reaches the low-latitude box, $i$ ts surface albedo $\alpha_{S L}$ is given by

$$
=\frac{a_{L} x_{S}+b\left(0.5-x_{S}\right)+s_{2}\left[-3 b / 16+\left(b-a_{L}\right)\left(x_{S}-x_{S}{ }^{3}\right) / 2\right]}{0.5-3 S_{2} / 16} \text {. }
$$

For both cases, the sine of the ice-edge latitude $x_{s}$ is determined by

$$
x_{S}=\left[\frac{2\left(T_{i}-T_{S}\right) / T_{2}+1}{3}\right]^{1 / 2} .
$$

In the above equations $b(=0.63)$ is the ice-covered surface albedo, and its value was chosen to be consistent with satellite observations (Coakley 1979). The constants $a_{H}(=0.230)$ and $a_{L}(=0.0873)$ are, respectively, the high- and low-latitude box icefree-surface albedos, evaluated by averaging over the ocean and land aibedos. Following the usual procedure, the ice-edge position is associated with a spec if ied isotherm $T_{j}\left(=-9 \cdot 6^{\circ} \mathrm{C}\right)$.

We will first adjust the model climate by matching the present values of $\bar{T}_{s}, T_{2}$, the planetary albedo, the outgoing thermal flux and the ice-cap size, a necessary tuning process to obtain the socalled control run, or the simulated present climate.

The most significant improvement of the present HLRD model (over the one-dimensional VRC model) is the ability to simulate the tropospheric lapse rate. It is particularly encouraging to note that the tropospheric lapse rates at 0 to 30 and 30 to $90^{\circ} \mathrm{N}$ are calculated to be 6.1 and $4.8 \mathrm{~K} \mathrm{~km}^{-1}$, respectively, which are in good agreement with observed values of 6.1 and $5.1 \mathrm{~K} \mathrm{~km}^{-1}$ at the respective latitude zones. This feature is of critical importance for studying the interactions between the vertical and meridional heat fluxes and their effect on global stability.

3. RESULTS

To study the effect of dynamical heat fluxes and their feedbacks on global stability, we ran the model with and without these feedbacks. For example, fixed vertical heat transport (VHT) means the vertical dynamical heat flux is fixed to be that of the control case (present climate) during cl imate change. Note that fixed VHT is different from the commonlyused fixed critical lapse-rate treatment, which still allows the VHT feedback.

As it has been pointed out by several authors (e.g. Cess and Wronka 1979, Warren and Schneider 1979, Endal and Schatten 1982) that the meridional heat transport parameterization has a crucial influence on the ice extent, we investigate the changes of the total (ocean and atmosphere) meridional heat flux and ice cover in response to the decreases in the solar constant.

For this purpose, starting from the present equilibrium climate (the control case), the model was run with gradually reduced (i.e. no large perturbations were applied to any equilibrium climate) solar constant values until a totally ice-covered Earth occurred. Such a quasistatic perturbation treatment was intended to allow the feedback mechanisms inherent in the climate system to act smoothly, and thus to el iminate (if any) possible spurious solutions (see North and others 1981).

Figure 1 shows the model-calculated ice-line 


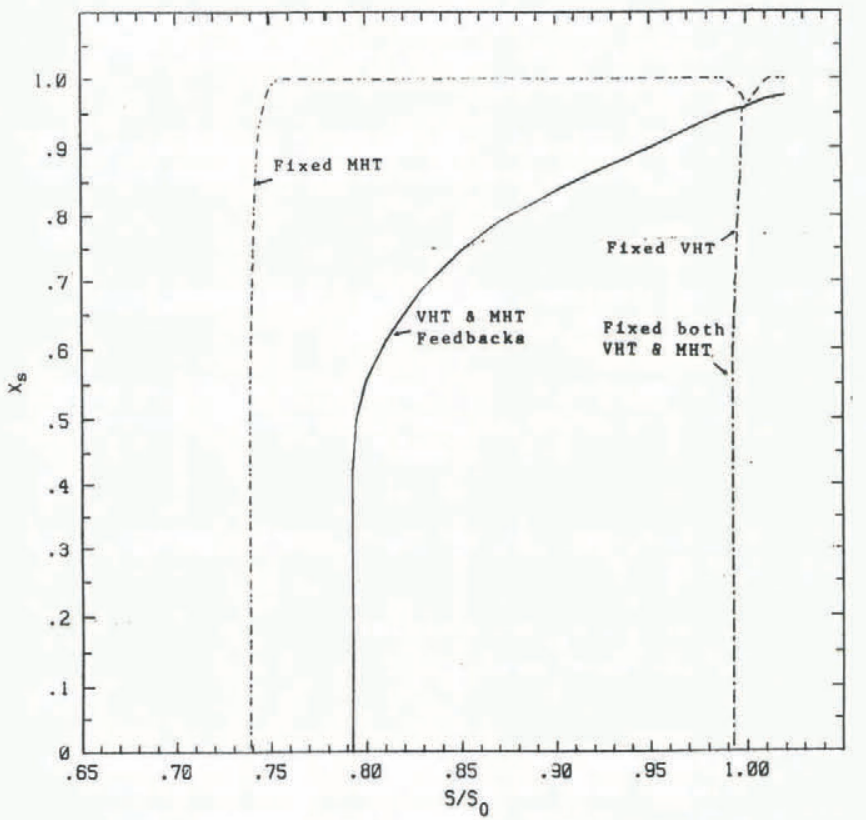

Fig.1. Feedback effects of meridional and vertical heat transport (MHT and VHT, respectively) on the ice-line position for solar constant change. Four scenarios were studied: fixed VHT, fixed both VHT and MHT, VHT and MHT feedbacks, and fixed MHT. The results for the former two scenarios are almost

identical and thus are represented by the same curve.

position as a function of the solar constant change. In the ZEB models, an unstable equilibrium state can be calculated (defined by $\left.d x_{s} / d\left(S / S_{0}\right)<0\right)$. However, in the HLRD model, the corresponding unstable state cannot be reached because of the use of a timemarching procedure. Consequently, the results of the unstable transitions from our model are indicated by vertical lines.
To study the dynamical heat-flux feedback, we have performed four model calculations: fixed VHT (case 1), fixed both VHT and MHT (case 2), both VHT and MHT feedbacks (case 3 ) and fixed MHT (case 4). Note, however, that fixed dynamical heat transport scenarios are used only for illustrative purposes.

Because the results for cases 1 and 2 are almost identical, both are represented by the same curve in Figure 1. The calculated global stability for case 3 (considered to be the most realistic one of the four cases) is $21 \%$, which is in sharp contrast with the $\sim 1 \%$ value obtained for cases 1 and 2 . The results suggest that the vertical heat-flux feedback is a strong negative feedback during climate change. Case 4 (fixed MHT) reveals some interesting features. When the MHT feedback was switched off, other feedback mechanisms, for example, the lapse rate, interacted with each other in such a way that a $26 \%$ solar constant reduction was needed for a total freeze. Moreover, these solutions indicate that the Earth is ice-free for most of its history. Note that these results are also in sharp contrast with the calculations for case 3 , in which the MHT feedback is included. In that case, the ice-cap exists even when the change of MHT is small (see Equation ( 3 ) and $T_{2}$ values in Table I). The implication is that the model climate is very sensitive to the presence or absence of MHT feedback. In fact, the model's current climate is unstable in the latter case. This kind of behavior for fixed MHT has been pointed out by Stone (in press). In addition, Barron and others (1981) have also found that if the present-day value of the meridional heat transport could be maintained (despite the decrease of the meridional temperature gradient) the nominally high Cretaceous temperatures (i.e. an ice-free Earth) can be achieved with a one-dimensional ZEB model.

Table I presents comparisons between cases 3 and 4 of the changes of other model cl imate parameters. These comparisons show that the difference between the model behavior for the two cases can be attributed to the different responses of the vertical dynamical heat flux and the baroclinic lapse rate. The results also suggest that fixed MHT results in a

TABLE I. EFFECTS OF TREATMENTS OF MERIDIONAL HEAT TRANSPORT (MHT) ON CHANGE OF MODEL CLIMATE DUE TO SOLAR CONSTANT DECREASE. IN BOTH TREATMENTS, THE VERTICAL HEAT-TRANSPORT FEEDBACK IS INCLUDED.

Decrease of solar constant (\%)

Parameter

$\begin{array}{ccccc}2 & 5 & 10 & 15 & 20 \\ -1.8 & -4.6 & -9.2 & -15.1 & -26.7 \\ 0.1 & -1.7 & -4.9 & -8.4 & -13.7 \\ \sim 0 & \sim 0 & \sim 0 & \sim 0 & 4 \\ 5 & 7 & 10 & 15 & 20\end{array}$

3. Vertical heat transport $\left(\mathrm{Wm}^{-2}\right)$

\begin{tabular}{|c|c|c|c|c|c|c|}
\hline \multirow{2}{*}{ A. $0-30^{\circ} \mathrm{N}$} & MHT feedback & -7.6 & -18.6 & -35.0 & -48.9 & -65.8 \\
\hline & fixed MHT & -7.4 & -18.1 & -34.3 & -46.6 & -61.6 \\
\hline \multirow{2}{*}{ B. $30-90^{\circ} \mathrm{N}$} & MHT feedback & -4.7 & -11.3 & -21.4 & -35.7 & -60.1 \\
\hline & I fixed MHT & -2.1 & -7.3 & -15.5 & -23.0 & -28.4 \\
\hline
\end{tabular}

4. Lapse rate $\left(K \mathrm{~km}^{-1}\right)$

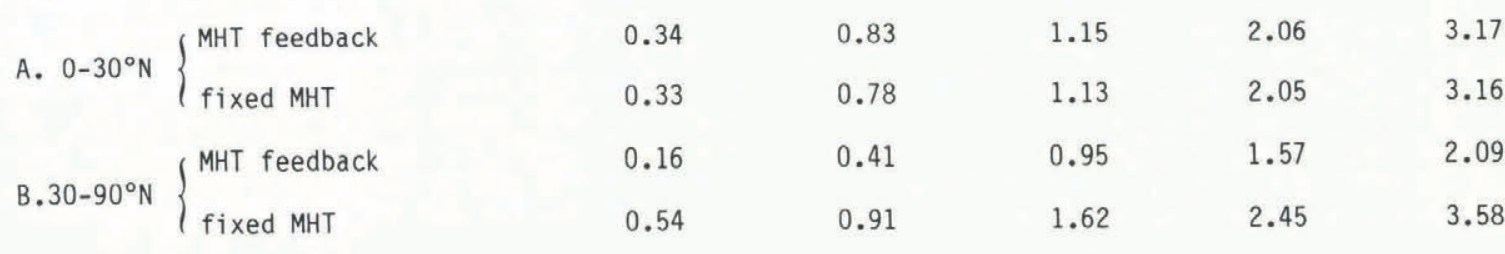


significantly smaller meridional temperature gradient while for MHT feedback, T2 maintains more or less a constant value, a feature also cited in Wang and Stone (1980).

Another interesting point is that for both cases the tropopause height decreased when solar constant was reduced and reached $\sim 400$ to 500 mbar at the limiting value of global stability. Similar behavior was reported by Wetherald and Manabe (1975), who obtained a tropopause height of 500 mbar for their white Earth experiment. In our model simulations with MHT feedbacks, the oceanic fraction was assumed to be $50 \%$ of the total (the atmosphere contains the other $50 \%$ ) before the ice 1 ine reaches $30^{\circ}$, and assumed zero thereafter (the atmosphere contains all the MHT). It was also found that keeping the ocean transport fixed (the same as the control experiment) led to a smaller global stability of $\sim 18.5 \%$.

\section{SUMMARY AND DISCUSSION}

We have examined the faint young sun-climate paradox using a coupled high- and low-latitude radiative-dynamical model. The model computes solar and thermal radiation and, more importantly, considers meridional and vertical dynamical heat-flux feedbacks. The results indicate that by including these feedback mechanisms, the model climate becomes much more stable than previous model results have indicated. Therefore we argue that no deus ex machina assumptions are needed to explain the faint young suncl imate paradox. For example, an enormous amount of $\mathrm{CO}_{2}$ is apparently not required to provide the greenhouse effect during the second half of the Earth's $h$ istory. This can be regarded as a very important consequence, since more recent findings (see Schatten and Endal 1982, Crowley 1983 for details) point out that no great amounts of greenhouse gases were present during the last $2 \mathrm{Ga}$. The model results suggest that the climate feedback mechanisms alone, combined perhaps with moderately increased concentrations of greenhouse gases and/or lower surface albedos, as well as increased oceanic heat transport, could have maintained not just a higher global stability, but a relatively ice-free Earth on the geological time scales.

However, we must emphasize that since the dynamical heat-flux parameterizations were derived on a bas is of the present $\mathrm{cl}$ imate conditions, they may not necessarily be valid when the climate is significantly different from that existing now. Moreover, the simple ice-edge conditions used for these experiments may be inadequate, especially when the ice line moves to low latitudes where the seasonal surface temperature variation is small.

In summary, much more work is needed in the context of global stability to develop more appropriate ice- 1 ine conditions, to parameterize oceanic heat transport and to construct surface albedo parameterization on geological time scales corresponding to the evolution of the 1 ithosphere (Hargraves 1976). In addition, model improvements, such as interactive clouds, cumulus convective parameterization for the tropics and seasonal variation should also be pursued. These parameterizations together with an improved knowledge of the chemical evolution of the Earth's atmosphere may bring us one step further to the understanding of the paradox.

\section{ACKNOWLEDGEMENT}

This research was supported by IJS National Science Foundation grant no. ATM-8204019.

\section{REFERENCES}

Barron E J, Thompson S L, Schneider S H 1981 An ice-free Cretaceous? Results from cl imate model simulations. Science 212(4494): 501-508

Budyko M I 1969 The effect of solar radiation variations on the climate of the Earth. Tellus 21(5): 611-619
Cess R D, Wronka J C 1979 Ice ages and the Milankovitch theory: a study of interactive climate feedback mechanisms. Tellus 31(3): 185-192

Coakley J A Jr 1979 A study of climate sensitivity using a simple energy balance climate model. Jour nal of the Atmospheric Sciences 35: 260-269

Crowley T J 1983 The geologic record of climatic change. Reviews of Geophysics and Space Physics 21: 828-877

Endal A S, Schatten K H 1982 The faint young sunclimate paradox: continental influences. Joumal of Geophysical Research 87: 7295-7302

Hargraves R B 1976 Precambrian geologic history. Science 193: 363-371

Hart MH 1978 The evolution of the atmosphere of the Earth. Ieame 33: 23-29

Kasting J F 1982 Stability of ammonia in the primitive terrestrial atmosphere. Joumal of Geophysical Research 87: 3091-3098

Knauth L P, Epstein S 1976 Hydrogen and oxygen isotope ratios in nodular and bedded cherts. Geochimica et Cosmochimica Acta 40: 1095-1108

Kuhn W R, Atreya S K 1979 Ammonia photolys is and the greenhouse effect in the primordial atmosphere of the Earth. Icarus 37: 207-213

Lacis A A, Hansen J E 1974 A parameterization for the absorption of solar radiation in the Earth's atmosphere. Jourmal of the Atmospheric Sciences 31: 118-133

Lindzen R S, Farrell B 1977 Some realistic modifications on simple climate models. Journal of the Atmospheric Sciences 34: 1487-1501

Lorenz E N 1979 Forced and free variation of weather and $\mathrm{Climate.} \mathrm{Joumal} \mathrm{of} \mathrm{the} \mathrm{Atmospheric}$ Sciences 36: 1367-1376

Manabe S, Strickler R F 1964 Thermal equilibrium of the atmosphere with a convective adjustment. Jour nal of the Atmospheric Sciences 21: 361-385

Newkirk G Jr 1980 Solar luminosity on time scales of $10^{5}$ years to $10^{9.6}$ years. Geochimica et Cosmochimica Acta. Supplement 13: 293-320

North G R 1975 Analytical solution to a simple climate model with diffusive heat transport. Joumal of the Atmosphemic Sciences 32: 1301-1307

North G R, Cahalan R F, Coakley J A Jr 1981 Energy balance $\mathrm{cl}$ imate models. Reviews of Geophysics and Space Physics 16: 465-489

Owen T, Cess R D, Ramanathan V 1979 Enhanced $\mathrm{CO}_{2}$ greenhouse to compensate for reduced solar luminosity on early Earth. Nature 277: 640-642

Sagan C, Mullen G 1972 Earth and Mars: evolution of atmosphere and surface temperatures. Science 177: $52-56$

Schatten K H, Endal A S 1982 The faint young sunclimate paradox: volcanic influences. Geophysical Research Letters 9(12): 1309-1311

Sellers W D 1969 A global climate model based on the energy balance of the Earth-atmosphere system. Journal of Applied Meteorology 8: 392-400

Stone $\mathrm{PH}$ In press Feedbacks between dynamical heat fluxes and temperature structure in the atmosphere. Fifth Biennial Ewing Symposium, 1983. Proceedings

Stone P H, Carlson J H 1979 Atmospheric lapse rate regimes and their parameterization. Joumal of the Atmosphemic Sciences 36: 415-423

Stone P H, Miller D A 1980 Empirical relations between seasonal changes in meridional temperature gradients and meridional fluxes of heat. Joumal of the Atmospheric Sciences 37: 1708-1721

Tarling D H 1978 The geological-geophysical framework of ice ages. In Gribbin J (ed) Climatic change. Cambridge etc, Cambridge University Press: 3-24

Wang W-C 1983 Effects of approximate radiation treatments used in the climate models on the clear sky thermal radiation flux and its perturbation due to $\mathrm{CO}_{2}$ increase. United States. Department of Energy. Technical Report DOE/ER/60023-1 
Wang W-C, Stone P H 1980 Effect of ice-albedo feedback on global sensitivity in a one-dimensional radiative-convective climate model. Joumal of the Atmospheric Sciences 37(3): 545-552

Wang $W-C$, Pinto J P, Yung Y L 1980 Climatic effects due to halogenated compounds in the Earth's atmosphere. Jourmal of the Atmospheric Sciences 37: 333-338

Wang W-C, Rossow W B, Yao M S, Wolfson M 1981 Climate sensitivity of a one-dimensional radiativeconvective model with cloud feedback. Jourmal of the Atmospheric Sciences 38: 1167-1178

warren S G, Schneider S H 1979 Seasonal simulation as a test for uncertainties in the parameterizations of a Budyko-Sellers zonal cl imate model. Joumal of the Atmospheric Sciences 36: 1377-1391

Wetherald R T, Manabe S 1975 The effects of changing solar constant on the climate of a general circulation model. Journal of the Atmospheric Sciences 32: 2044-2059 